\title{
Dissolution of a Colloidal Particle in an Oscillatory Flow
}

\author{
Dezhuang Ye, ${ }^{1}$ Robin Bogner, ${ }^{2}$ Ji-Qin Li, ${ }^{1}$ and Tai-Hsi Fan*1 \\ ${ }^{1}$ Mechanical Engineering, University of Connecticut, Storrs, CT 06269-3139, USA \\ ${ }^{2}$ Pharmaceutical Sciences, University of Connecticut, Storrs, CT 06269-3139, USA
}

(Dated: August 22, 2016)

Understanding dissolution kinetics of a colloidal particle in an aqueous solution is of great importance in many pharmaceutical and biochemical applications. We present theoretical analysis of low Reynolds number flow dynamics and mass transfer of a dissolving spherical particle in a unidirectional oscillatory flow field. The coupling of fluid flow and passive motion of the particle are solved analytically, and the transient mass transfer associated with the oscillation of the particle is computed numerically. The flow patterns, basic characteristics of mass transport, and the simplified long-time dissolution process under various saturation concentrations and relative flow conditions are discussed in details.

Keywords: Dissolution kinetics, oscillatory flow, colloidal particle, mass transfer, pharmaceutical 


\section{INTRODUCTION}

Better understanding of dissolution of a colloidal particle to the surrounding liquid environment is of great importance in broad industrial applications. There exist lots of relevant chemical processes in liquid-liquid, solid-liquid, and gas-liquid systems ranging from absorbtion, extraction, leaching, drying, crystallization, dissolution, to combustion of solid and liquid fuels [1-9]. In pharmaceutical sciences, investigations on the reconstitution of various pharmaceutical composition and dosage forms based on dissolution methods are essential for practical purposes [10-13]. Specifically, therapeutic molecules such as monoclonal antibodies are often highly concentrated to reduce the volume injected into the patient. The concentrated protein therapeutics are lyophilized or freeze-dried from a solution in a vial, producing a porous plug or cake in order to improve storage stability. Just prior to administration to the patient, the cake is reconstituted with sterile water or other aqueous diluent. However, reconstitution of these highly concentrated protein therapeutics is a challenge, due to unacceptably long and variable reconstitution times. The methods of reconstitution (i.e., the rate and location to add the sterile diluent, and the fluid flow mixing pattern) [14-17] are known to affect the time required for reconstitution. A typical and preferred method of mixing is by moving the vial with a horizontal orbital motion (Figs. 1a and 1b). An end-over-end or other vigorous shaking method is less often used due to the generation of fresh air/water interfaces at which proteins may denature. Under the most common orbital shaking mode, a free surface wave driven by the gravity and centrifugal effects travels circumferentially, which introduces a locally periodic oscillation pattern within the fluid. Figures 1c and 1d illustrate the linear approximation of the first two eigenmodes of the free surface wave and a typical oscillatory flow pattern beneath the wave, respectively. A suspending colloidal particle within the fluid thus will move passively by following the periodic flow while its mass is dissolving into the medium.

Quantitative analysis of dissolution mechanisms remains a great scientific challenge. Complicated dissolution scenario often involves disintegration of various drug dosage forms, transient flow field, and multiphase transport phenomena. The dissolving particle, particle clump, or cake interacts with the surrounding fluid medium, and the process can be further complicated by the physicochemical properties, size and shape, and packing density of the particles to be dissolved. The basic diffusive and convective transport phenomena 
(a) orbital shaking

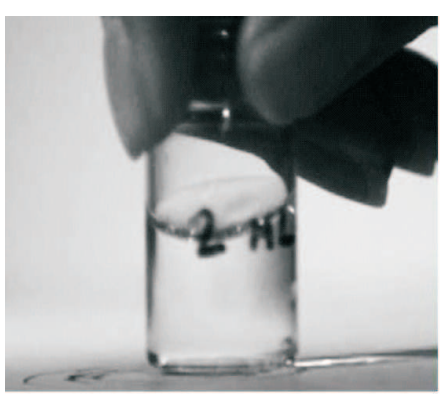

(c) free surface wave

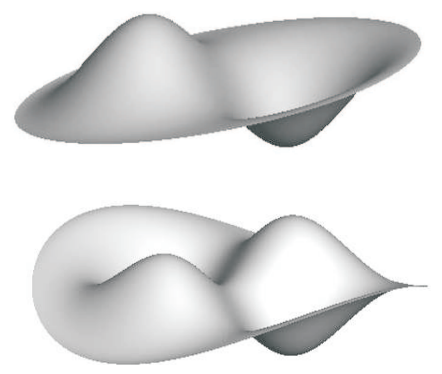

(b) top view

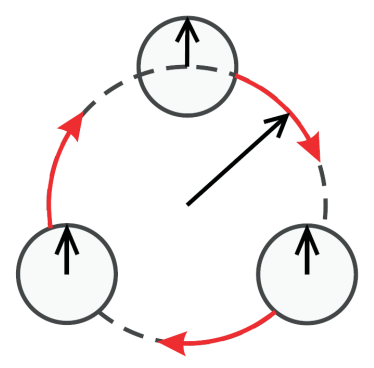

(d) oscillatory flow

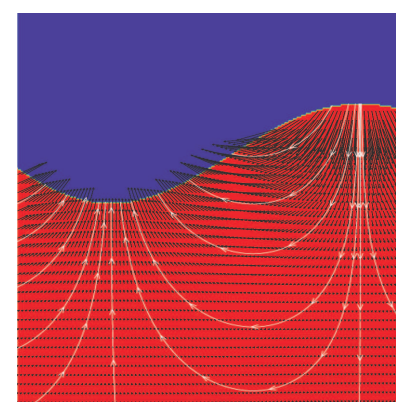

FIG. 1: (a) A typical vial used for protein reconstitution by manual shaking, the vial size is about $1 \mathrm{~cm}$ in diameter and $2 \mathrm{~cm}$ in height, (b) a top view of the orbital motion while the vial itself does not rotate, (c) the first two eigenmodes of a simplified free surface wave, and (d) the oscillatory flow beneath the wave.

therefore play important roles in dispersing the drugs and in determining the efficiency of reconstitution. However, both the primary and secondary flows involved in a more realistic, three-dimensional and periodic fluid motion is fairly complicated. Here we simplify the flow pattern to a unidirectional oscillatory flow in the low particle Reynolds number regime and focus on the investigation of local behaviors of the dissolution process. Under this theoretical assumption, the particle suspended in fluid moves passively back and forth at the same frequency as the external flow but with a phase lag. The particle's inertia along with the flow conditions determine the amplitudes of the displacement and velocity of the particle and the phase lag of its motion relative to the flow. Intuitively we know that relative motion between the particle and the flow promotes the convective mass transfer and enhances the overall dissolution rate. A quantitative analysis is essential in determining the baseline dissolution efficiency. Early investigations on the correlation of enhanced heat or mass transfer 
have focused on steady flow under various strength of convective flows [18-25]. Others are on a steady acoustic streaming flow in which the nonlinear inertial effect dominates [26-29]. In this paper we focus on the time dependency of mass transfer characteristics driven by a monochromatic oscillatory flow. The particle size and the relative velocity between the flow and the particle are in the low Reynolds number regime so that the nonlinear inertial effect of the flow is negligible. The transient dissolution of a solid colloidal particle has a much longer time scale than momentum transport so that the coupling of flow dynamics and the particle motion can be resolved analytically based on a quasi-steady approximation of the moving boundary condition. The coupling of mass transfer and long-time dissolution kinetics are numerically computed for various saturation concentration, flow strength, and driving frequency.

\section{THEORETICAL ANALYSIS}

Figure 2 illustrates the problem in hand, a spherical colloidal particle (presumable in the colloidal regime and the particle size is less than $1 \mathrm{~mm}$, much smaller than the vial size) is undergoing a single-frequency periodic translational motion driven by an oscillatory external flow. The assumed far field velocity is $U \mathrm{e}^{-i \omega t} \hat{\mathbf{e}}_{x}$, and the particle velocity has an assumed

phase lag relative to the flow, $\tilde{U}_{\mathrm{p}} \mathrm{e}^{-i \omega t} \hat{\mathbf{e}}_{x}$, where $U$ is a real amplitude and $\tilde{U}_{\mathrm{p}}$ is a complex amplitude that accommodates the phase lag $\phi$. The phase lag for the periodic motion is to be found based on the simplified linear momentum equation. The axisymmetric coordinates in radial $r$ and polar $\theta$ directions define the position in the field, and $x$ indicates the axial direction along which the flow and particle oscillate. The periodic flow and passive particle motion are on a relatively short time scale compared to the long time dissolution process. We therefore apply a quasi-steady moving boundary for the dissolution interface to facilitate the analytical solution. The particle size or moving interface is applied to the momentum and mass transfer explicitly from the computed dissolution kinetics.

\section{A. Flow Dynamics}

Assuming that the oscillatory flow is in the Stokes regime (e.g., particle radius less than $1 \mathrm{~mm}$, kinematic viscosity of the aqueous solution about $10^{-6} \mathrm{~m}^{2} / \mathrm{s}$, and characteristic flow 


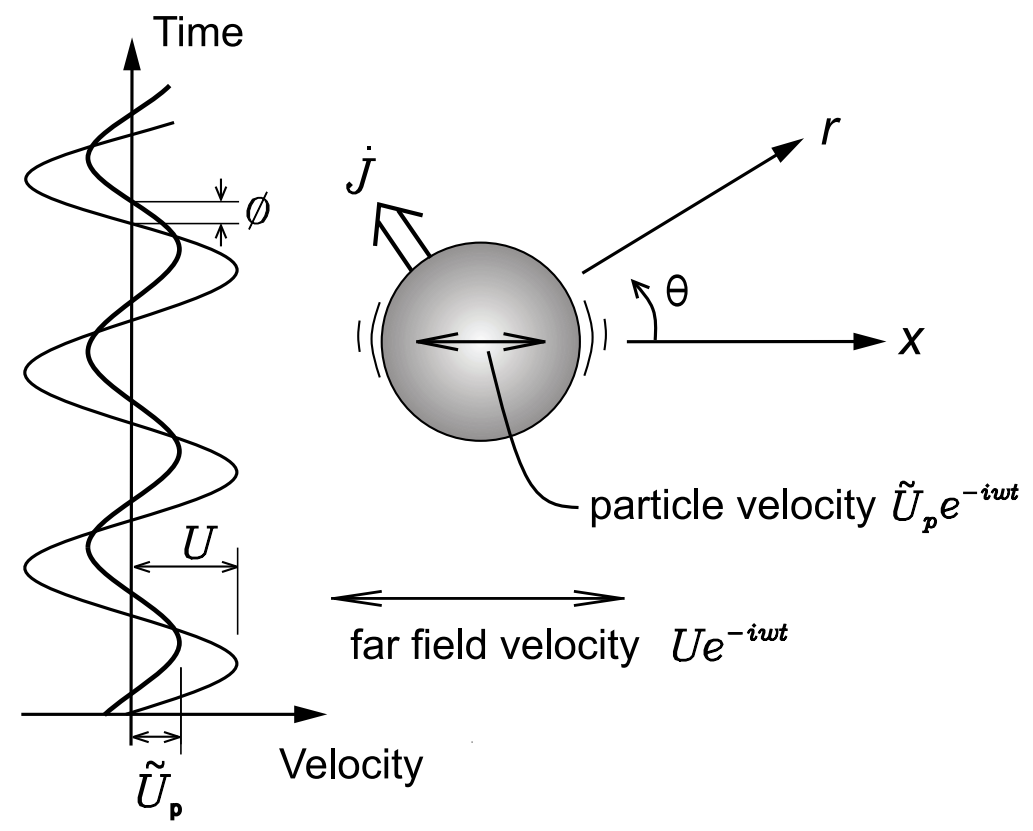

FIG. 2: Schematic of a colloidal particle under passive motion driven by an oscillatory flow. The mass flux $\dot{\boldsymbol{J}}$ is due to surface dissolution. The phase angle $\phi$, later defined in Eq. (36), represents the lag of particle motion and the driving oscillatory flow.

velocity less than $1 \mathrm{~mm} / \mathrm{s}$ ). The linear viscous flow for a Newtonian fluid is governed by the transient Stokes equation:

$$
\rho \frac{\partial \boldsymbol{v}}{\partial t} \simeq-\nabla p+\eta \nabla^{2} \boldsymbol{v}
$$

for a periodic motion within the fluid domain $R\left(t_{\mathrm{L}}\right) \leq r \leq \infty$ and $0 \leq \theta<\pi$, where $t_{\mathrm{L}}$ indicates the long time scale, $R\left(t_{\mathrm{L}}\right)$ is the particle radius, assumed constant during transient fluid motion, $\boldsymbol{v}$ is flow velocity, $p$ is pressure, $t$ is time, $\rho$ is fluid density, and $\eta$ is the dynamic viscosity. Note that we use $t$ to present the short time dynamics and transient diffusion, and $t_{\mathrm{L}}$ to represent a long time dissolution process. The incompressible continuity equation is $\nabla \cdot \boldsymbol{v}=0$. In the laboratory coordinate system, the corresponding pressure and velocity boundary conditions can be defined as

$$
\begin{gathered}
p \rightarrow p_{\infty}=\tilde{p}_{\infty} \mathrm{e}^{-i \omega t}+p_{0} \quad \text { as } \quad r \rightarrow \infty \\
\boldsymbol{v} \rightarrow \boldsymbol{v}_{\infty}=U \mathrm{e}^{-i \omega t} \hat{\mathbf{e}}_{x} \quad \text { as } \quad r \rightarrow \infty
\end{gathered}
$$

and

$$
\boldsymbol{v}=\boldsymbol{v}_{\mathrm{p}}=\tilde{U}_{\mathrm{p}} \mathrm{e}^{-i \omega t} \hat{\mathbf{e}}_{x} \quad \text { at } \quad r=R\left(t_{\mathrm{L}}\right),
$$


where the subscript $p$ indicates particle, $U$ is the velocity amplitude at far field, the tilde $\sim$ emphasizes a complex amplitude that carries the phase lag information, $\hat{\mathbf{e}}_{x}$ is the unit vector along the axial direction, $\omega$ is circular frequency, and $R\left(t_{\mathrm{L}}\right)$ is the particle radius under quasi-steady approximation. To simplify the formulation, we consider the following relative velocity field to the far-field condition:

$$
\boldsymbol{v}_{\mathrm{rel}}=\boldsymbol{v}-\boldsymbol{v}_{\infty}
$$

and thus the transient Stokes equation can be written as

$$
\rho\left(\frac{\partial \boldsymbol{v}_{\mathrm{rel}}}{\partial t}+\frac{\partial \boldsymbol{v}_{\infty}}{\partial t}\right) \simeq-\nabla p+\eta \nabla^{2} \boldsymbol{v}_{\mathrm{rel}}
$$

Now by defining

$$
p_{\text {rel }}=p-p_{\infty}+p_{0}
$$

we obtain

$$
\nabla p_{\text {rel }}=\nabla p-\nabla p_{\infty}=\nabla p+\rho \frac{\partial \boldsymbol{v}_{\infty}}{\partial t} .
$$

Combining Eqs. (6) and (8), the transient Stokes system on the relative coordinate system can be simplified and expressed as

$$
\begin{gathered}
\rho \frac{\partial \boldsymbol{v}_{\mathrm{rel}}}{\partial t} \simeq-\nabla p_{\text {rel }}+\eta \nabla^{2} \boldsymbol{v}_{\mathrm{rel}}, \\
p_{\text {rel }} \rightarrow p_{0} \quad \text { as } \quad r \rightarrow \infty, \\
\boldsymbol{v}_{\mathrm{rel}}=\boldsymbol{v}_{\mathrm{p}}-\boldsymbol{v}_{\infty} \quad \text { at } \quad r=R\left(t_{\mathrm{L}}\right), \\
\boldsymbol{v}_{\mathrm{rel}} \rightarrow 0 \quad \text { as } \quad r \rightarrow \infty .
\end{gathered}
$$

The instantaneous total force acting on the moving particle results in an acceleration of the particle. The equation of motion for the particle thus can be expressed as

$$
\frac{4}{3} \rho_{\mathrm{p}} \pi R^{3} \frac{d \boldsymbol{v}_{\mathrm{p}}}{d t}=\mathcal{F} \hat{\mathbf{e}}_{x}=\int_{\partial \Omega} \boldsymbol{\tau} \cdot \hat{\mathbf{e}}_{r} d A
$$

where $\rho_{\mathrm{p}}$ is particle mass density, $\boldsymbol{\tau}$ is flow stress, $\hat{\mathbf{e}}_{r}$ is unit vector in radial direction and the outward normal along the particle surface, and the area integration of the traction applies to the particle surface $\partial \Omega$. We now look for the flow field $\boldsymbol{v}_{\text {rel }}$ and particle's instantaneous velocity, acceleration and position. 
To better characterize the coupling of dynamics and mass transfer, we consider typical characteristic scales for the length, time, velocity, stress and pressure, and the total hydrodynamic drag force as follows:

$$
\begin{aligned}
& r \sim R\left(t_{\mathrm{L}}\right), \quad t \sim 1 / \omega, \quad \boldsymbol{v} \sim U \\
& \boldsymbol{\tau} \text { and } p \sim \eta U / R, \quad \text { Drag } \mathcal{F} \sim \eta R U .
\end{aligned}
$$

Hereafter all variables and formulations are in dimensionless forms unless further notification. By substituting the location- and time-dependent periodic velocity and pressure field, i.e.,

$$
\left[\boldsymbol{v}_{\text {rel }}(\mathbf{r}, t), p_{\text {rel }}(\mathbf{r}, t)\right]=\left[\tilde{\boldsymbol{v}}_{\text {rel }}(\mathbf{r}), \tilde{p}_{\text {rel }}(\mathbf{r})\right] \mathrm{e}^{-i t}+\left[0, p_{0}\right]
$$

into Eqs. (9) to (13), with tilde indicating the complex amplitudes of the transient fields and $\mathbf{r}$ representing the position vector, we obtain

$$
\begin{gathered}
-i \beta \tilde{\boldsymbol{v}}_{\mathrm{rel}}=-\nabla \tilde{p}_{\mathrm{rel}}+\nabla^{2} \tilde{\boldsymbol{v}}_{\text {rel }}, \\
\tilde{p}_{\mathrm{rel}} \rightarrow 0 \quad \text { as } \quad r \rightarrow \infty, \\
\tilde{\boldsymbol{v}}_{\mathrm{rel}}=\left(\tilde{U}_{\mathrm{p}}-1\right) \hat{\mathbf{e}}_{x} \quad \text { at } r=1, \\
\tilde{\boldsymbol{v}}_{\mathrm{rel}} \rightarrow 0 \quad \text { as } \quad r \rightarrow \infty,
\end{gathered}
$$

and

$$
\frac{4}{3} \pi \lambda \beta \frac{d \boldsymbol{v}_{\mathrm{p}}}{d t}=\mathcal{F} \hat{\mathbf{e}}_{x}=\int_{\partial \Omega} \boldsymbol{\tau} \cdot \hat{\mathbf{e}}_{r} d A
$$

where the two resulting dimensionless numbers are defined as

$$
\beta=\frac{\rho \omega R^{2}\left(t_{L}\right)}{\eta} \text { and } \quad \lambda=\frac{\rho_{\mathrm{p}}}{\rho}
$$

The frequency parameter $\beta$ compares the local acceleration to the viscous effects, and the inertial factor $\lambda$ is the particle-to-fluid density ratio that measures how closely the particle follows the fluid motion.

We apply Landau's pseudo vector approach for solving the transient Stokes flow problems [30]. By incorporating the passive motion of the sphere we obtain the flow field and particle velocity simultaneously. First by taking curl of the momentum equation to eliminate the pressure term, and expressing the complex velocity amplitude as

$$
\tilde{\boldsymbol{v}}_{\mathrm{rel}}=\left(\tilde{U}_{\mathrm{p}}-1\right) \nabla \times \nabla \times\left(f \hat{\mathbf{e}}_{x}\right)
$$


where $f$ is a radially dependent function to be determined, the momentum equation reduces to

$$
\nabla^{2} \nabla^{2} \nabla f+i \beta \nabla^{2} \nabla f=0
$$

Integrating the above momentum equation and considering the quiescent relative far-field condition, the radial function $f$ thus satisfies

$$
\nabla^{2} \nabla^{2} f+k^{2} \nabla^{2} f=0
$$

where the characteristic wave number is

$$
k(\beta)=(1+i) \sqrt{\frac{\beta}{2}}=\frac{(1+i)}{\delta(\beta)} .
$$

The wave number is associated with the viscous penetration depth $\delta=\sqrt{2 / \beta}$, which is inversely proportional to the oscillation frequency and often used to describe the influence of the diffusive viscous wave that propagates into the fluid medium. At higher frequency and lower fluid viscosity the depth reduces. The above 4th-order differential equation and corresponding boundary conditions for the unknown radial function $f$ is analytically solvable. The relative velocity amplitude in radial and polar directions therefore are given by

$$
\begin{gathered}
\tilde{v}_{\mathrm{r}}^{\mathrm{rel}}=\left(\tilde{U}_{\mathrm{p}}-1\right) \cos \theta\left[\frac{2 c_{1} \mathrm{e}^{i k r}}{r^{2}}\left(\frac{1}{i k r}-1\right)-\frac{2 c_{2}}{r^{3}}\right], \\
\tilde{v}_{\theta}^{\mathrm{rel}}=-\left(\tilde{U}_{\mathrm{p}}-1\right) \sin \theta\left[\frac{c_{1} \mathrm{e}^{i k r}}{r}\left(\frac{1}{r}-\frac{1}{i k r^{2}}-i k\right)+\frac{c_{2}}{r^{3}}\right],
\end{gathered}
$$

where the coefficients $c_{1}$ and $c_{2}$ are

$$
c_{1}=-\frac{3}{2 i k} \mathrm{e}^{-i k}, \quad c_{2}=-\frac{1}{2}\left(1-\frac{3}{i k}-\frac{3}{k^{2}}\right) .
$$

The corresponding amplitude of the relative pressure field can be written as

$$
\tilde{p}_{\text {rel }}=\frac{c_{2}\left(\tilde{U}_{\mathrm{p}}-1\right) k^{2}}{r^{2}} \cos \theta,
$$

and the amplitudes of the deviatoric stress field are

$$
\begin{gathered}
\tilde{\sigma}_{\mathrm{rr}}=4\left(\tilde{U}_{\mathrm{p}}-1\right) \cos \theta\left[\frac{3 c_{2}}{r^{4}}+\frac{c_{1} \mathrm{e}^{i k r}}{r^{4}}\left(3 r-\frac{3}{i k}-i k r^{2}\right)\right], \\
\tilde{\sigma}_{\mathrm{r} \theta}=-\left(\tilde{U}_{\mathrm{p}}-1\right) \sin \theta \times \\
{\left[-\frac{6 c_{2}}{r^{4}}+\frac{c_{1} \mathrm{e}^{i k r}}{r^{4}}\left(-6 r+\frac{6}{i k}+3 i k r^{2}+k^{2} r^{3}\right)\right] .}
\end{gathered}
$$


Substituting the velocity field and completing the area integration of the surface force, Eq. (20) results in

$$
\begin{aligned}
& \mathcal{F} \hat{\mathbf{e}}_{x}=\frac{4}{3} \pi \lambda \beta \frac{d \boldsymbol{v}_{\mathrm{p}}}{d t}=-\frac{4}{3} \pi \lambda k^{2} \tilde{U}_{\mathrm{p}} \mathrm{e}^{-i t} \hat{\mathbf{e}}_{x} \\
&=6 \pi\left(1-\tilde{U}_{\mathrm{p}}\right)\left(1-i k-\frac{1}{9} k^{2}\right) \mathrm{e}^{-i t} \hat{\mathbf{e}}_{x}
\end{aligned}
$$

where the three terms included in the second bracket on the right can be treated as the force contributions from the Stokes drag, penetration of the viscous wave, and virtual or added mass of the sphere. When $k \rightarrow 0$ the result reduces a typical Stokes drag and the particle has approximately zero or fully relaxed acceleration. Rearranging the above equation results in the velocity amplitude of the particle:

$$
\tilde{U}_{\mathrm{p}}(k, \lambda)=\frac{1-i k-k^{2} / 9}{1-i k-(1+2 \lambda) k^{2} / 9} .
$$

Up to this point a few typical limiting cases can be validated. For a particle with small density, $\lambda \ll 1, \tilde{U}_{\mathrm{p}} \rightarrow 1$, particle inertia is negligible and its motion follows the driving oscillatory flow. For $\lambda \gg 1, \tilde{U}_{\mathrm{p}} \rightarrow 0$, particle essentially remains stationary and this condition can be used to describe a fixed and dissolving particle in a oscillatory flow. At very low oscillation frequency, $\omega \rightarrow 0, \beta$ and thus $k \rightarrow 0$, and therefore $\tilde{U}_{\mathrm{p}} \rightarrow 1$. This is equivalent to zero inertial effect such that the relative motion is fully relaxed and the particle follows the flow completely. At large oscillation frequency, $k \gg 1$, the inertial effect becomes significant and $\tilde{U}_{\mathrm{p}} \rightarrow 1 /(1+2 \lambda)$.

Finally, the real velocity and trajectory of the particle can be extracted from the complex amplitude, expressed as

$$
\tilde{U}_{\mathrm{p}}=U_{\mathrm{p}} \mathrm{e}^{-i \phi}
$$

where $\phi$ represents the phase lag compared to the driving oscillatory flow. With few algebraic operations, the explicit forms that describe the particle's motion can be expressed as

$$
\begin{aligned}
U_{\mathrm{p}}(\lambda, \beta) & =\frac{A_{1}}{\sin \phi+A_{1} \cos \phi-A_{2} \sin \phi}, \\
\phi(\lambda, \beta) & =\tan ^{-1}\left(\frac{A_{1}}{A_{1}^{2}-A_{2}+A_{2}^{2}}\right),
\end{aligned}
$$

where the parameters

$$
A_{1}=-\frac{9}{2 \lambda \beta}\left(1+\frac{\sqrt{2 \beta}}{2}\right)
$$


and

$$
A_{2}=-\frac{1}{2 \lambda \beta}\left(1+\frac{9}{\sqrt{2 \beta}}\right) .
$$

Accordingly, the real part of the force acting on the particle becomes

$$
\begin{aligned}
\mathcal{R}(\mathcal{F})= & 6 \pi\left(1+\frac{1}{\delta}\right)\left[\cos t-U_{\mathrm{p}} \cos (t+\phi)\right] \\
& -6 \pi\left(\frac{1}{\delta}+\frac{2}{9 \delta^{2}}\right)\left[\sin t-U_{\mathrm{p}} \sin (t+\phi)\right] .
\end{aligned}
$$

The force can also be separated to two parts that are proportional to the far-field velocity and acceleration relative to the particle, expressed here as a dimensional form:

$$
\begin{aligned}
\mathcal{F} \hat{\mathbf{e}}_{x}= & 6 \pi \eta R\left(1+\frac{R}{\delta}\right)\left(\boldsymbol{v}_{\infty}-\boldsymbol{v}_{\mathrm{p}}\right) \\
& +6 \pi \eta R\left(\frac{\rho R^{2}}{9 \eta}+\frac{R}{\omega \delta}\right) \frac{d\left(\boldsymbol{v}_{\infty}-\boldsymbol{v}_{\mathrm{p}}\right)}{d t} .
\end{aligned}
$$

It is observed from above equation that when velocity magnitude remains the same and frequency increases, the penetration depth $\delta$ decreases, acceleration of fluid is suppressed, and the viscous dissipation eventually dominates the dynamic behavior. During the orbital shaking process, however, the velocity magnitude would likely increase as the frequency increase due to similar displacement. Next we present the mass transfer model that underlies the simplified spherical dissolution process.

\section{B. Mass transfer and dissolution kinetics}

In the laboratory coordinates, the single species transport equation can be written as

$$
\frac{\partial c}{\partial t}+\boldsymbol{v} \cdot \nabla c=D \nabla^{2} c
$$

where $c$ is the transient, local concentration of the dissolving substance, and $D$ is the assumed constant diffusivity in the aqueous solution. To simplify the moving boundary problem, we consider Galilean transformation of the laboratory coordinates (unprimed) to the moving coordinates (primed) with origin affixed to the center of the particle. For a unidirectional motion in Cartesian coordinates, we consider

$$
x^{\prime}=x-\int \boldsymbol{v}_{\mathrm{p}}(t) d t, \quad y^{\prime}=y, \quad z^{\prime}=z
$$


and time $t^{\prime}=t$, the transformed time derivative in $x$-direction becomes

$$
\frac{\partial}{\partial t} \equiv \frac{\partial}{\partial t^{\prime}}+\frac{\partial}{\partial x^{\prime}} \frac{\partial x^{\prime}}{\partial t} \equiv \frac{\partial}{\partial t^{\prime}}-\boldsymbol{v}_{\mathrm{p}} \frac{\partial}{\partial x^{\prime}}
$$

Therefore Eq. (41) can be written as

$$
\frac{\partial c}{\partial t^{\prime}}-\boldsymbol{v}_{\mathrm{p}} \cdot \nabla^{\prime} c+\boldsymbol{v} \cdot \nabla^{\prime} c=D \nabla^{\prime 2} c
$$

or equivalently,

$$
\frac{\partial c}{\partial t^{\prime}}+\boldsymbol{v}^{\prime} \cdot \nabla^{\prime} c=D \nabla^{\prime 2} c
$$

where $\boldsymbol{v}^{\prime}$ here is the relative velocity of the flow field to the moving particle. Note that the previous relative velocity $\boldsymbol{v}_{\text {rel }}$ is defined differently as the flow field relative to the far-field boundary condition. The complementary initial and boundary conditions are

$$
\begin{gathered}
c\left(r^{\prime}, 0\right)=0 \text { for } \quad R_{0} \leq r^{\prime}<\infty, \\
c\left(R\left(t_{\mathrm{L}}\right), t^{\prime}\right)=c_{0} \quad \text { for } \quad t^{\prime}>0,
\end{gathered}
$$

and

$$
c \rightarrow 0 \quad \text { as } \quad r^{\prime} \rightarrow \infty
$$

respectively, where $c_{0}$ is the assumed saturation concentration of the dissolving molecules of interest in the aqueous phase, and $R_{0}$ is the initial particle radius at $t_{\mathrm{L}}=t^{\prime}=0$. To couple the oscillatory flow and the convective diffusion equation one can better characterize the mass transfer by applying the following scales:

$$
r \sim R_{0}, \quad t^{\prime} \sim 1 / \omega, \quad \boldsymbol{v}^{\prime} \sim U, \quad c^{\prime} \sim c_{0}
$$

The scaled transport equation thus can be expressed as

$$
\beta S_{\mathrm{c}} \frac{\partial c}{\partial t^{\prime}}+\frac{1}{2} P_{\mathrm{e}} \boldsymbol{v}^{\prime} \cdot \nabla^{\prime} c=\nabla^{\prime 2} c
$$

where the corresponding initial and boundary conditions are $c=0$ at $t^{\prime}=0, c=1$ at $r=R\left(t_{\mathrm{L}}\right) / R_{0}$, and $c \rightarrow 0$ as $r \rightarrow \infty$. The three characteristic numbers are the frequency parameter $\beta$, the Schmidt number $S_{\mathrm{c}}$, and the Péclet number $P_{\mathrm{e}}$ based on the bulk flow velocity, defined as

$$
\beta=\frac{\rho \omega R_{0}^{2}}{\eta}, \quad S_{\mathrm{c}}=\frac{\nu}{D}=\frac{\mu}{\rho D}, \quad P_{\mathrm{e}}=\frac{2 R_{0} U}{D}
$$


where $\nu$ is the kinematic viscosity. The analytical relative velocity field can be obtained from

$$
\boldsymbol{v}^{\prime}=\boldsymbol{v}_{\mathrm{rel}}+\boldsymbol{v}_{\infty}-\boldsymbol{v}_{\mathrm{p}}
$$

From Eqs. (3), (4), (26), (27), and by taking the real part of the components of the complex velocity field in radius and polar directions, we obtain

$$
\begin{aligned}
& v_{r}^{\prime}=\left[\cos t^{\prime}-U_{\mathrm{p}}(\lambda, \beta) \cos \left(\phi(\lambda, \beta)+t^{\prime}\right)\right] \cos \theta \times \\
& \left\{\frac { 3 R ( t _ { \mathrm { L } } ) } { r ^ { 2 } } \mathrm { e } ^ { ( R - r ) \sqrt { \beta / 2 } } \left[\sqrt{\frac{1}{2 \beta}} \cos \left(\sqrt{\frac{\beta}{2}}(r-R)\right)\right.\right. \\
& \left.-\left(\frac{1}{r \beta}+\sqrt{\frac{1}{2 \beta}}\right) \sin \left(\sqrt{\frac{\beta}{2}}(r-R)\right)\right] \\
& \left.\quad-\frac{R^{3}}{r^{3}}\left(1+\frac{1}{R} \sqrt{\frac{9}{2 \beta}}\right)+1\right\} \\
& +\left[\sin t^{\prime}-U_{\mathrm{p}} \sin \left(\phi+t^{\prime}\right)\right] \cos \theta \times \\
& \left\{\frac { 3 R } { r ^ { 2 } } \mathrm { e } ^ { ( R - r ) } \sqrt { \beta / 2 } \left[\left(\frac{1}{r \beta}+\sqrt{\frac{1}{2 \beta}}\right) \cos \left(\sqrt{\frac{\beta}{2}}(r-R)\right)\right.\right. \\
& \left.\left.\quad+\sqrt{\frac{1}{2 \beta}} \sin \left(\sqrt{\frac{\beta}{2}}(r-R)\right)\right]-\frac{3 R^{3}}{r^{3}}\left(\frac{1}{R \beta}+\sqrt{\frac{1}{2 \beta}}\right)\right\}
\end{aligned}
$$


and

$$
\begin{aligned}
& v_{\theta}^{\prime}=\left[\cos t^{\prime}-U_{\mathrm{p}}(\lambda, \beta) \cos \left(\phi(\lambda, \beta)+t^{\prime}\right)\right] \sin \theta \times \\
& \left\{\frac { 3 R ( t _ { L } ) } { 2 r ^ { 3 } } \mathrm { e } ^ { ( R - r ) \sqrt { \beta / 2 } } \left[\left(r^{2}+r \sqrt{\frac{1}{2 \beta}}\right) \times\right.\right. \\
& \cos \left(\sqrt{\frac{\beta}{2}}(r-R)\right)-\left(\frac{1}{\beta}+r \sqrt{\frac{1}{2 \beta}}\right) \times \\
& \left.\left.\sin \left(\sqrt{\frac{\beta}{2}}(r-R)\right)\right]-\frac{R^{3}}{2 r^{3}}\left(1+\frac{1}{R} \sqrt{\frac{9}{2 \beta}}\right)-1\right\} \\
& +\left[\sin t^{\prime}-U_{\mathrm{p}} \sin \left(\phi+t^{\prime}\right)\right] \sin \theta \times \\
& \left\{\frac { 3 R } { 2 r ^ { 3 } } \mathrm { e } ^ { ( R - r ) \sqrt { \beta / 2 } } \left[\left(\frac{1}{\beta}+r \sqrt{\frac{1}{2 \beta}}\right) \cos \left(\sqrt{\frac{\beta}{2}}(r-R)\right)\right.\right. \\
& \left.+\left(r^{2}+r \sqrt{\frac{1}{2 \beta}}\right) \sin \left(\sqrt{\frac{\beta}{2}}(r-R)\right)\right] \\
& \left.-\frac{3 R^{3}}{2 r^{3}}\left(\frac{1}{R^{2} \beta}+\frac{1}{R} \sqrt{\frac{1}{2 \beta}}\right)\right\}
\end{aligned}
$$

respectively, where the phase functions $\phi=\phi(\lambda, \beta)$ and amplitude of the particle velocity $U_{\mathrm{p}}=U_{\mathrm{p}}(\lambda, \beta)$ are given by Eqs. (36) and (35), respectively. The particle size $R\left(t_{\mathrm{L}}\right)$ is resolved by the dissolution kinetics described below.

Once the mass transfer equation is computed, dissolution kinetics in the long time regime can be resolved by tracing the time variation of the particle radius $R\left(t_{\mathrm{L}}\right)$. In principle the mass flux distribution is non-uniform around the particle surface, and the flux may depend on the local stress acting on it. These concerns complicate the problem significantly. To simplify the dissolution kinetics we assume that the particle remains spherical throughout the dissolution process and the particle size is determined by the diffusive-based local flux in an average sense. That is, the small blowing velocity [31, 32] due to density variation at the interface is neglected. Now by considering the first-order kinetics, the time rate of change of total mass dissolved in the aqueous solution can be expressed as

$$
\frac{d m}{d t_{\mathrm{L}}}=\left.\int_{\partial \Omega} D \frac{\partial c}{\partial r}\right|_{r=R\left(t_{\mathrm{L}}\right)} d S,
$$

where $m=4 \rho_{p} \pi R^{3}\left(t_{\mathrm{L}}\right) / 3$ is the particle mass, $t_{\mathrm{L}}$ is the time variable for the long-time dissolution process, and $R\left(t_{\mathrm{L}}\right)$ represents an effective radius of the particle. In terms of 
polar spherical coordinates, the above equation can be expressed as

$$
\rho_{\mathrm{p}} \frac{d R\left(t_{\mathrm{L}}\right)}{d t_{\mathrm{L}}}=\left.\frac{1}{2} \int_{0}^{\pi} D \frac{\partial c}{\partial r}\right|_{r=R\left(t_{\mathrm{L}}\right)} \sin \theta d \theta
$$

and thus the dissolution kinetics can be characterized by the time scale,

$$
\tau_{\mathrm{d}}=\frac{\rho_{\mathrm{p}} R_{0}^{2}}{D c_{0}}
$$

where $R_{0}$ is the initial radius of the particle, and $c_{0}$ is the saturation concentration. By comparing the long- to short-time scales, we have

$$
\frac{\tau_{\mathrm{d}}}{1 / \omega}=\beta S_{\mathrm{c}} \frac{\rho_{\mathrm{p}}}{c_{0}} \sim \mathcal{O}\left(10 \text { to } 10^{8}\right)
$$

where $\rho_{\mathrm{p}} / c_{0}$ is usually on the order of 1 to $10^{3}[33,34], S_{\mathrm{c}}$ is on the order of $10^{3}$, and for $\beta$ if we select a wide range of values from $10^{-2}$ to $10^{2}$, the dissolution characteristic time $\tau_{\mathrm{d}}$ is in general much larger than the short-time scale appears in the oscillatory flow dynamics and the mass transfer on redistribution of the species within an oscillation cycle. This justifies the quasi-steady assumption for the moving boundary in analyzing flow dynamics and mass transport, enabling us to decouple the computation of momentum/mass transport from the dissolution kinetics. The transport equation is computed by MacCormack's predictorcorrector scheme [35] along with the analytical velocity field.

\section{RESULTS AND DISCUSSIONS}

\section{A. Flow Dynamics}

Figure 3 shows instantaneous flow patterns around the moving particle in an oscillatory flow field. At nominal particle-to-fluid density ratio $\lambda=1$ and frequency parameter $\beta=10$ (e.g., $\omega=10 \mathrm{rad} / \mathrm{s}, R=10^{-3} \mathrm{~m}, \rho / \eta \simeq 10^{6} \mathrm{~s} / \mathrm{m}^{2}$ ), the interplay of the fluid flow and particle motion is significantly influenced by the inertial effects of both the fluid and the spherical particle. The maximum displacement of the particle in this case is about its radius. Nearly $3 / 8$ of a cycle is shown in Fig. 3, including the representative instants when flow speed reaches the maximum (3a), flow speed reduces significantly ( $3 \mathrm{~b}$ and $3 \mathrm{c}$ ), flow arrives at a reversal point but particle is not yet reversed ( $3 \mathrm{~d}$ and $3 \mathrm{e})$, and the instant when particle reverses its direction (3f). The linear transient flow exhibits interesting flow patterns. 

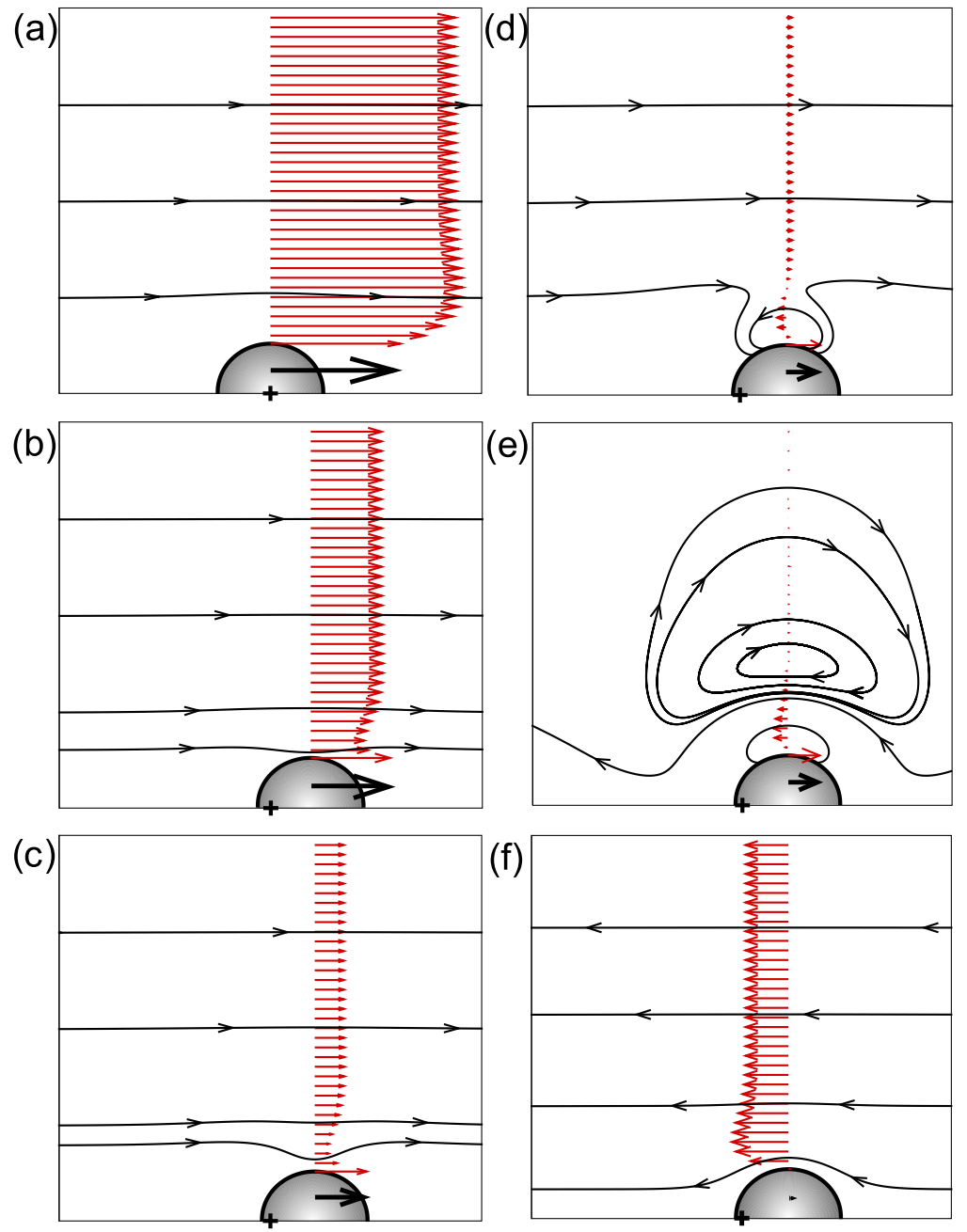

FIG. 3: Instantaneous flow patterns with streamlines around the particle and velocity vectors at $\theta=\pi / 2$ based on the laboratory coordinate system. The particle-to-fluid density ratio $\lambda=1$ and frequency parameter $\beta=10$. The sequential plots (a) to (f) are at time $t=0,0.1875 T, 0.225 T$, $0.245 T, 0.2625 T$, and $0.35 T$, respectively, where $T=2 \pi / \omega$ is the dimensional period. The arrows attached to the particle represent particle velocity.

Starting from the zero displacement point (Fig. 3a), the flow passing through the particle is quite uniform except a slight local acceleration near the particle surface. The velocity difference between particle and the flow reduces gradually to zero at the no-slip boundary on the particle surface. The deceleration of the oscillatory flow creates a momentum deficit near the particle surface $(3 \mathrm{~b})$ because the particle's inertial effect at this point leads to a higher velocity than the rest of the flow field. Apparently this momentum deficit provides a negative velocity gradient and thus the shear stress to slow down the particle motion. The 
toroidal vortex around the particle appears when flow decelerates significantly and about to reverse. While the inner and primary vortex is attached to the particle surface, the outer and much weaker secondary toroidal vortex appears (3e). The outer vortex expands, migrates, and dissipates quickly into the far field. The inner vortex is later suppressed when the bulk flow starts to reverse. A more uniform flow appears again at the instant that the sphere reverses its direction (3f). Although the toroidal vortex structure is of fundamental interest, it is expected that the weak circulation and short appearance time of both vortices have relatively less influence on the mass transfer. The particle's inertia or memory effect is more important in a way that it reduces the relative velocity of the flow to the particle, and thus suppresses convective mass transfer near the particle surface.

For the case with large particle inertia, $\lambda \gg 1$, the particle remains almost stationary, which is equivalent to a fixed particle located within a uniformly oscillating flow (Fig. 4). The analytical result is well established [30]. Experimental tests on the dissolution kinetics may be carried out by affixing a particle on a grid fixture in the vial, which will enhance the dissolution due a stronger convective effect. The sequential plots (a) to (f) shown in Fig. 4 are at the same time instants shown in Fig. 3 for comparison. For a fixed particle, the flow acceleration near the particle surface is more enhanced (4a and 4f), but only a single toroidal vortex is observed (4c to 4e). The vortex is first detached from the particle surface (4c), and then moving laterally, expanding, and finally dissipating into the far field.

In pharmaceutical practices, oscillating frequency is often a control parameter for the dissolution tests. Figure 5 shows the overall dynamic response of the particle to the oscillation frequency, particle size, fluid viscosity and density effects. Equation (35) provides the analytical result for the real part of the scaled velocity amplitude under various conditions in terms of $\beta$ and $\lambda$. The results are presented by self-similar curves. For higher $\lambda$ values, the particle has higher inertial resistance to the fluid motion and thus its absolute velocity reduces. When frequency $\omega \rightarrow 0$ and thus $\beta \rightarrow 0$, the fluid motion becomes quasi-steady and all inertial effects vanish to a degree such that the particle follows fluid motion completely. For a given density ratio, particle size, and aqueous medium, increasing oscillation frequency introduces higher inertial effects on both fluid and the particle. At higher frequency the overall force acting on the particle is enhanced but the particle's inertial effect dominates and its velocity reduces. Here a higher frequency can represent same displacement with higher flow velocity or same velocity but smaller displacement as long as the flow is within the low 
(a)

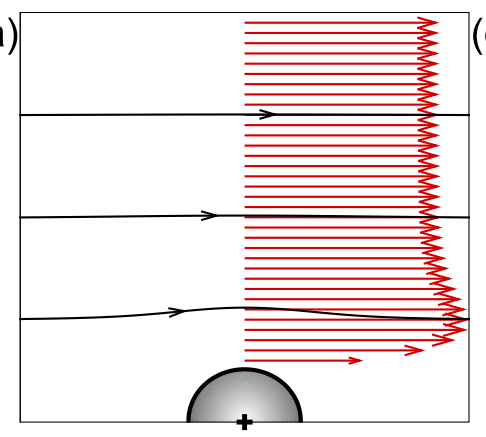

(b)

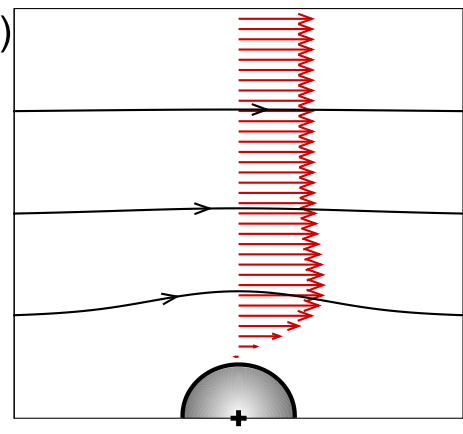

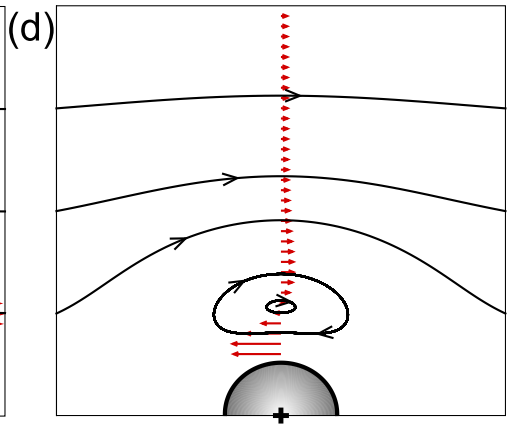

(e)

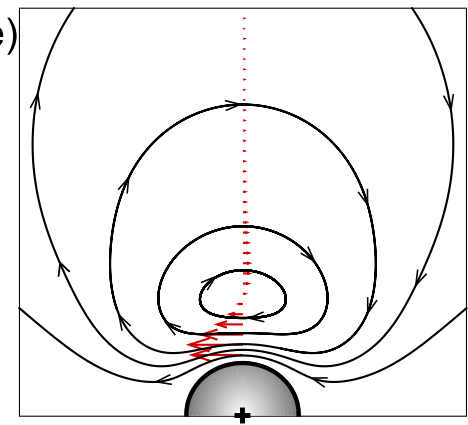

(c)
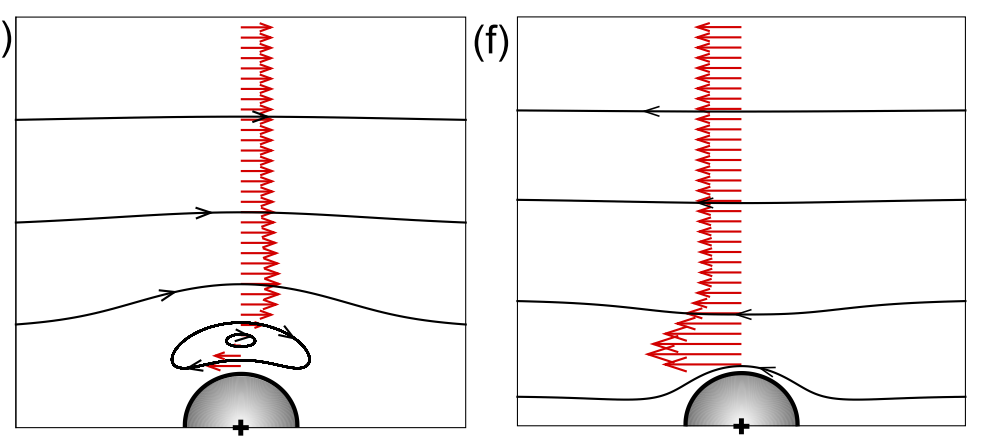

FIG. 4: Instantaneous flow patterns and passive particle motion at particle-to-fluid density ratio $\lambda \gg 1$ and frequency parameter $\beta=10$. The sequential plots (a) to (f) are at the same instants as shown in Fig. 3 .

Reynolds number regime. The lower bound for the velocity amplitude $U_{\mathrm{p}} \rightarrow 1 /(1+2 \lambda)$ as $\beta \rightarrow \infty$ is given by Eq. (32).

The time delay of the particle velocity $U_{p}$ corresponding to the hydrodynamic force is demonstrated in Fig. 6, showing transient results for a full cycle of oscillation. The circle points on the dashed line refer to the time instants shown in Fig. 3. The hydrodynamic force is determined by the stress based on the relative velocity field. The positive fluid-to-particle force and particle velocity indicate $+x$-direction. At $t=0$ the bulk flow reaches its maximum velocity and is larger than the particle velocity in any case of passive motion, resulting in positive force to accelerate the particle further until the particle's maximum speed is 


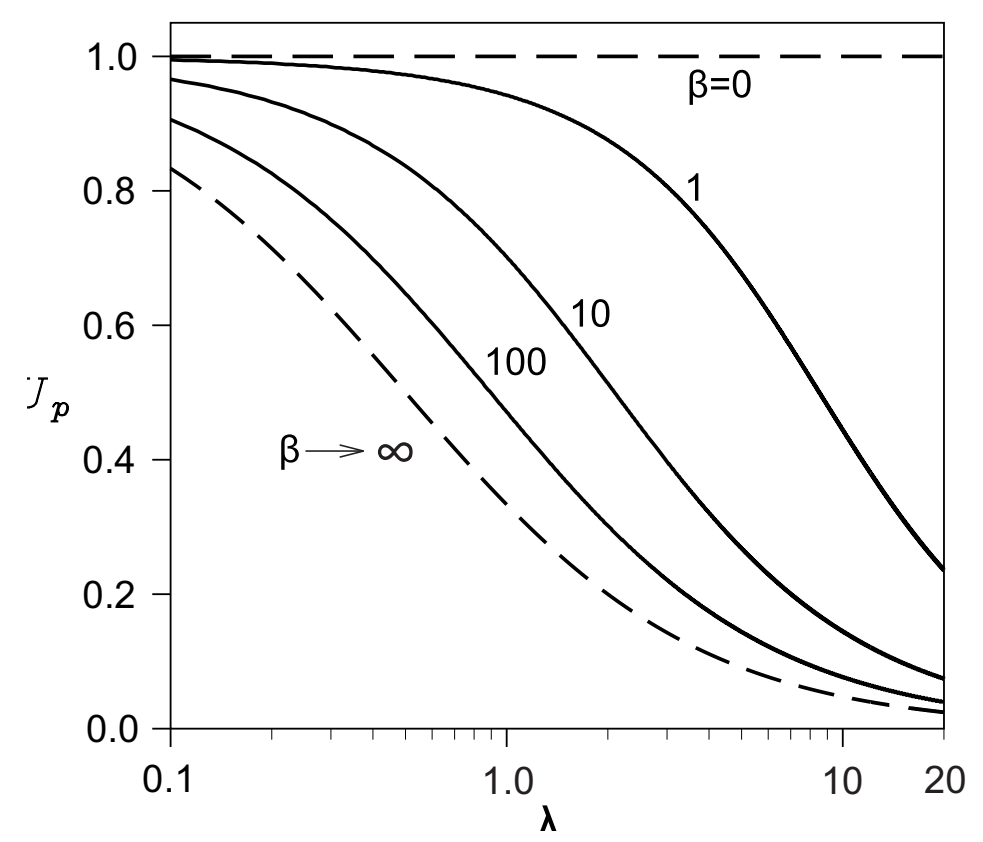

FIG. 5: The scaled amplitude of particle velocity $U_{p}$ versus particle-to-fluid density ratio $\lambda$ at various oscillation frequency.

achieved. After that the flow decelerates quickly while the force reduces and reverses, and later again the flow reaches its maximum speed. As seen in Fig. 3 for $\lambda=1$, a negative velocity gradient appears near the particle surface at instants (b) and (c), and the shear stress acts to slow down the particle motion. For a heavier particle, a larger overall hydrodynamic force is obtained due to higher relative velocity between the particle and the bulk flow, while the particle has lower absolute velocity $U_{\mathrm{p}}$. The influence of frequency is complicated as particle's inertia and both the viscous and inertial effects of the fluid are all involved. Under the same velocity amplitude of the bulk flow, a higher oscillation frequency leads to higher viscous and inertial forces on the particle but the the particle's acceleration is slightly reduced (Fig. 6). It is because the oscillation takes less time for the flow to accelerate or decelerate and turn around so that the particle appears somewhat caged and oscillates with a small amplitude near its mean position.

\section{B. Mass Transfer and Dissolution}

Dissolution kinetics is determined by the local mass flux around the spherical particle under various oscillation conditions. The scaled transport equation (50) shows that mass 

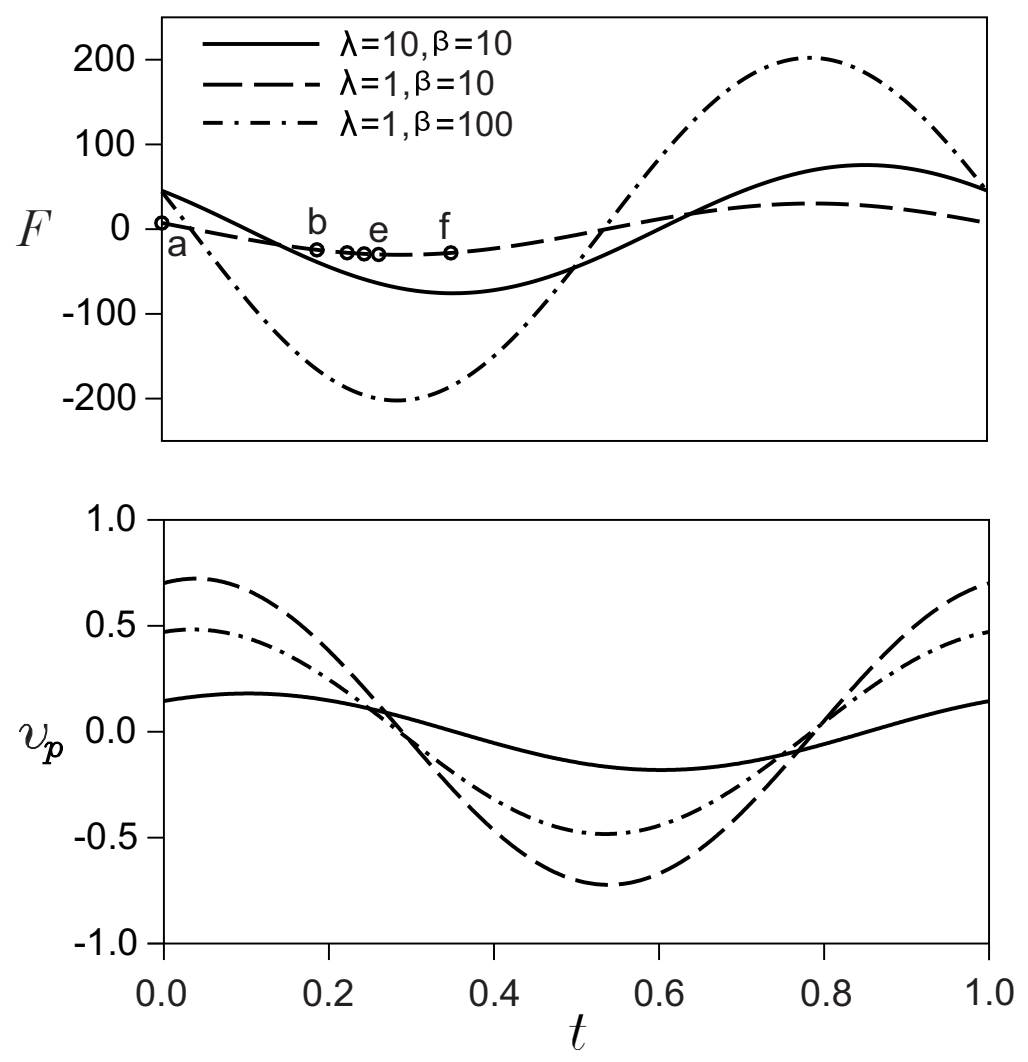

FIG. 6: Hydrodynamic force $\mathcal{F}$ (scaled by $\eta U R$ ) acting on the particle and the particle velocity $U_{\mathrm{p}}$ (scaled by the maximum bulk velocity $U$ ) versus time (scaled by $2 \pi / \omega$ ) in an oscillating flow field during a full cycle. Labels (a) to (f) indicate the time instants shown in Fig. 3.

transfer can be characterized by frequency parameter $\beta$, Schmidt number $S_{\mathrm{c}}$, and Péclet number $P_{\mathrm{e}}$. Considering an aqueous solution and a typical diffusivity of small molecules the Schmidt number $S_{\mathrm{c}}$ is around $10^{3}$. Here we discuss the influence of frequency parameter and Péclet number. Figure 7 shows the interplay of fluid flow and concentration field for $\beta=1$ and $P_{\mathrm{e}}=5000$ at several time instants. A concentration boundary layer is formed at the left fore front of the sphere when flow speed increases significantly. The boundary layer is accompanied by a steep concentration gradient along the particle surface, which enhances the local mass flux. This flux however is weakened near the rear part of the sphere. At later time the asymmetric distribution (7a) recovers to a more fore-and-aft symmetric pattern (7b) when flow reverses, and continues to form a temporary boundary layer near the right surface of the sphere (7c). If the bulk velocity remains the same, increasing oscillation frequency or reducing the displacement amplitude will enhance this recovery and thus have no significant promotion to the overall mass transfer. If the flow strength and frequency 


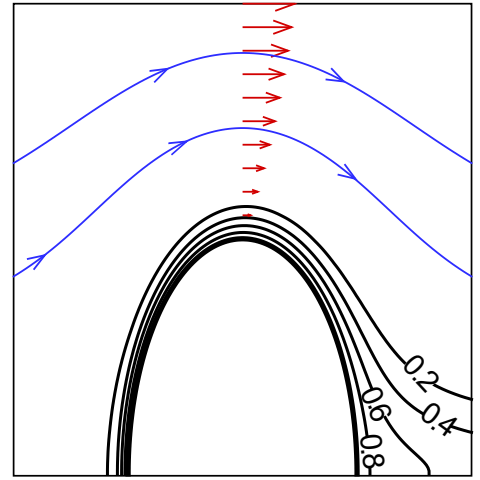

(a)

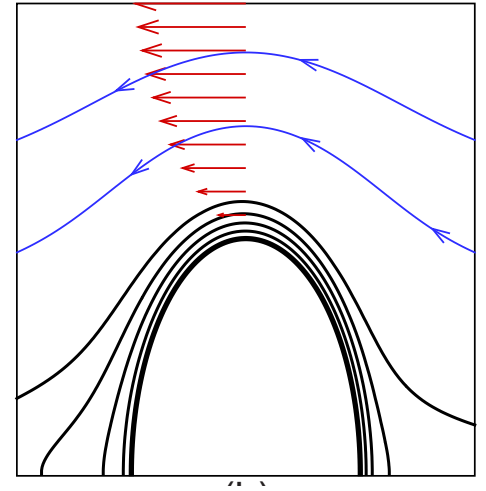

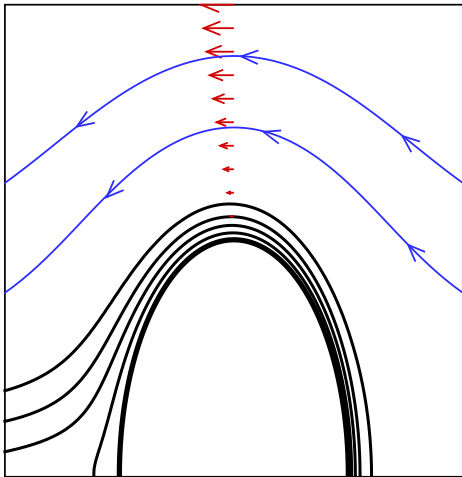

(c)

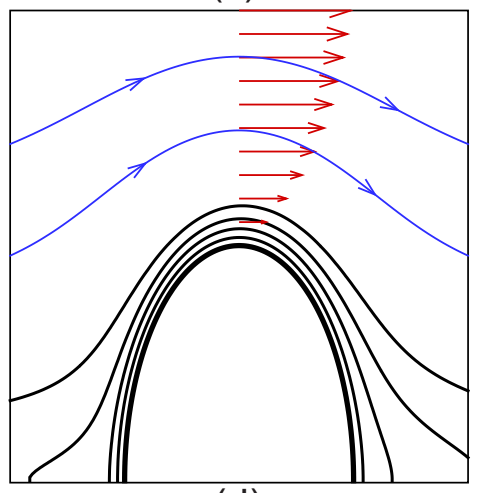

FIG. 7: Scaled concentration field showing the convective effect on the redistribution of molecular solutes around a sphere within an oscillatory flow field. The four concentration contours from 0.2 to 0.8 are shown at time instants $t=1.14$ (a), 1.45 (b), 1.67 (c), and 1.96 (d), scaled by $2 \pi / \omega$, and for $\beta=1$ and $P_{\mathrm{e}}=5000$.

increase proportionally, one would expect, in general, mass transfer enhances as Péclet number increases.

Once the concentration field is computed, the overall mass transfer rate can be quantified by the Sherwood number $S_{\mathrm{h}}$ as a comparison of total mass transfer contributed by convective and diffusive effects to the diffusion-only mass transfer rate. The averaged Sherwood number can be formulated as an area integration of surface diffusive flux around the axisymmetric sphere:

$$
S_{\mathrm{h}}=-\left.\frac{1}{2} \int_{0}^{\pi} \frac{\partial c}{\partial r}\right|_{r=1} \sin \theta d \theta .
$$

A transient correlation is not available for the problem in hand. As a validation we thus compare the numerical results with the established asymptotes for the steady state solu- 


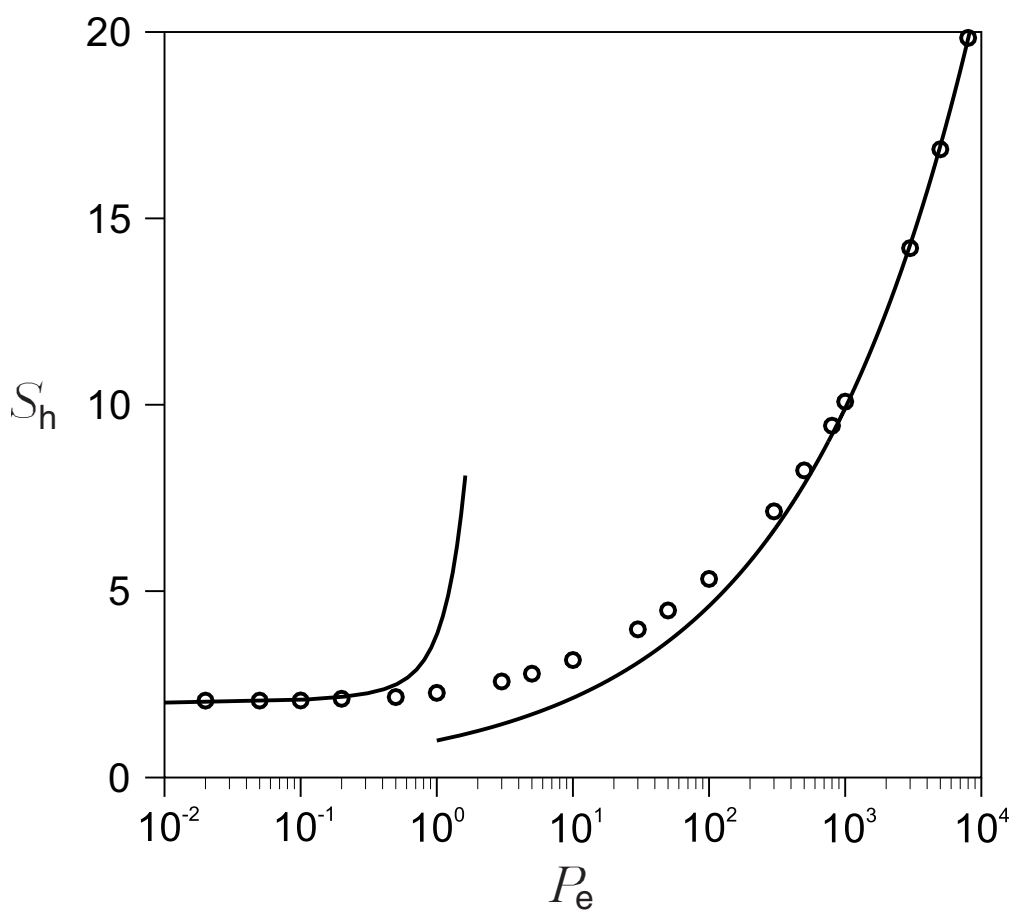

FIG. 8: Averaged Sherwood number versus Péclet number showing the enhancement of mass transfer under steady convection. The data points are current numerical results while the solid lines present asymptotic solutions, Eqs. (60) and (61).

tions $[18,19,22]$ :

$$
S_{\mathrm{h}}=2+\frac{1}{2} P_{\mathrm{e}}+\frac{581}{1920} P_{\mathrm{e}}^{2}+\cdots \quad \text { for } \quad P_{\mathrm{e}} \ll \mathcal{O}(1)
$$

and

$$
S_{\mathrm{h}} \rightarrow 0.991 P_{\mathrm{e}}^{\frac{1}{3}} \quad \text { as } \quad P_{\mathrm{e}} \rightarrow \infty
$$

where the Péclet number is defined as $P_{\mathrm{e}}=2 R_{0} U / D$. Figure 8 shows the consistency of numerical (data points) and analytical (solid curves) results. Mass transfer enhances monotonically as Péclet number increases, and this enhancement is also true for transient cases if the oscillation frequency remains the same.

Figure 9 shows the transient evolution of averaged Sherwood number near the initial stage of the dissolution. The results are presented for (a) a fixed particle with assumed constant size in an oscillating fluid at $\beta=1$ and $P_{\mathrm{e}}$ up to 5000, and (b) a freely suspended particle that moves passively with the flow at $\beta=1, P_{\mathrm{e}}=5000$, and $\lambda=1,5$, and 10. A large concentration gradient and thus the mass flux appears at the very beginning when the concentration is zero everywhere in the field and a saturation concentration $c_{0}$ is 
(a) fixed
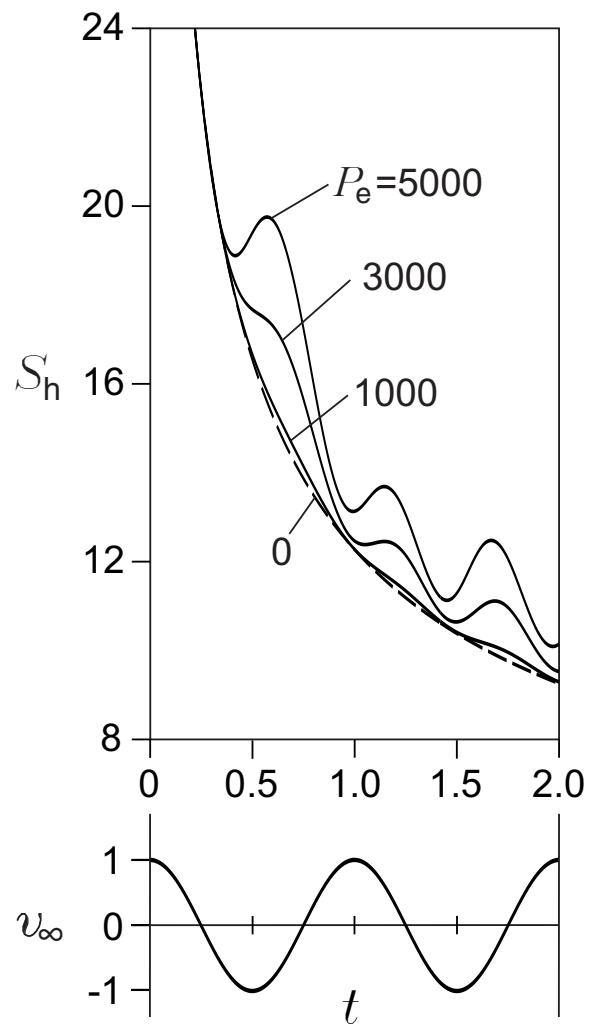

(b) free

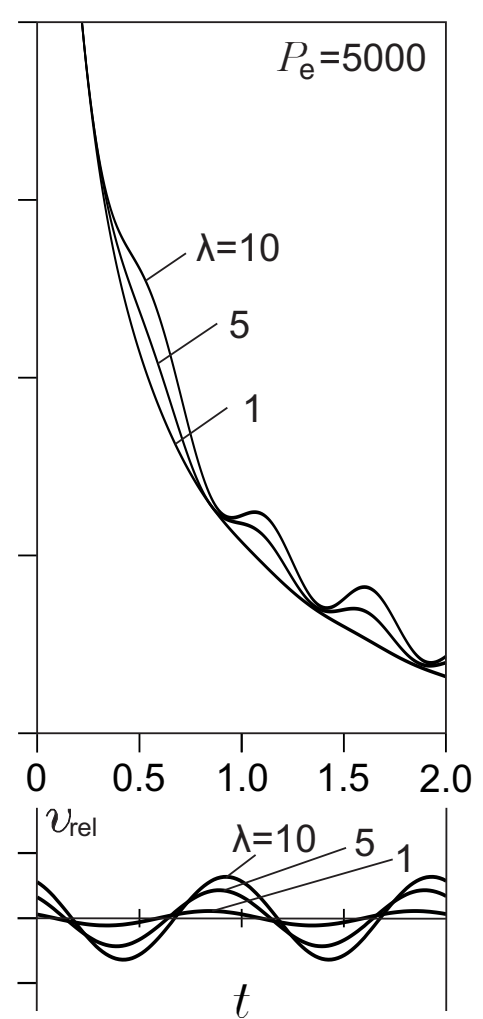

FIG. 9: Transient evolution of the mean Sherwood number in an oscillatory flow for (a) a fixed particle, $\beta=1$ and $P_{\mathrm{e}}=0,1000,3000$, and 5000, and (b) a freely suspended particle at $\beta=1$, $P_{\mathrm{e}}=5000, \lambda=1,5$, and 10 . The relative velocity at far-field is shown at the bottom of each figure, respectively. Time $t$ is scaled by $2 \pi / \omega$.

assigned to the colloid surface, which leads to a step concentration profile and high mass flux. The oscillatory nature of the transient Sherwood number comes from flow reversal and redistribution of the solute concentration. Strong convective effect appears as flow speed increases and a concentration boundary layer appears in front of the particle, which enhances the local mass flux during each half cycle. For a fixed particle (Fig. 9a) at $\beta=1$ and Péclet number less than 1000, the oscillatory nature of the Sherwood number is no longer noticeable. The transient relaxation eventually approaches a purely diffusive case $\left(P_{\mathrm{e}}=0\right)$. For a freely suspended particle, at lower inertia $\lambda=1$, the particle follows the flow more closely so that the relative velocity between particle and external flow reduces significantly, and the Sherwood number approaches a purely diffusive case without noticeable oscillatory behavior. For a case with higher particle inertia, e.g. $\lambda=5$ or $\lambda=10$, the enhancement 
of mass transfer and the oscillatory nature of the transient Sherwood number become more noticable at each half cycle. Note that the peak Sherwood number and flow velocity are not in phase. During each half cycle the peak of the Sherwood number is achieved a while after the speed starts to reduce from its maximum value (Fig. 9a and 9b).

(a) fixed

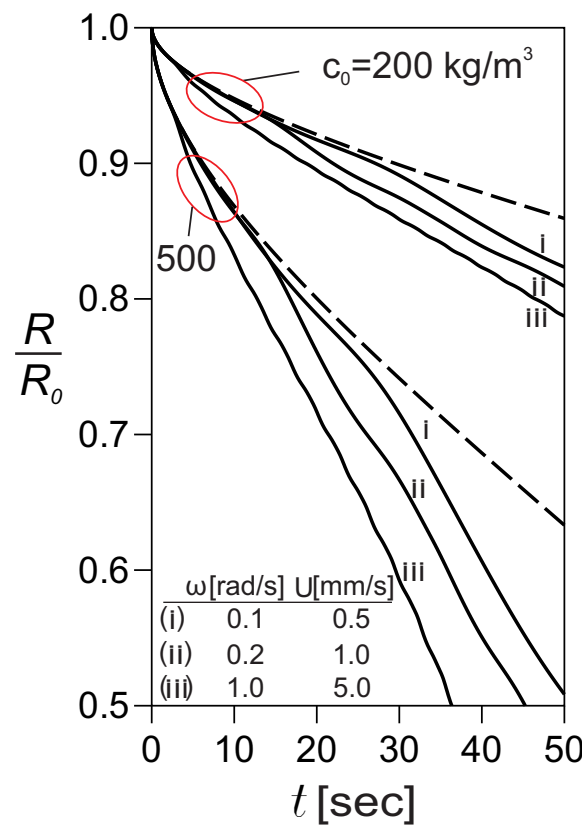

(b) free

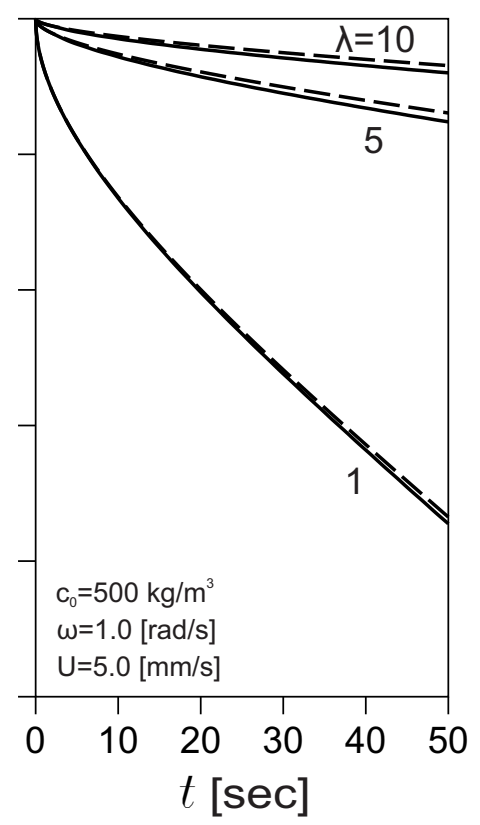

FIG. 10: (a) Transient evolution of the radius of a fixed and dissolving particle in an oscillatory flow under various driving frequency and bulk velocity with $\beta=1$ and $\lambda=1$. Two sets of results are presented for surface saturation concentrations, $c_{0}=200$ and $500 \mathrm{~kg} / \mathrm{m}^{3}$, respectively. Inertial effect on a freely suspended and dissolving particle in oscillatory flow at $\lambda=1,5$, and 10 . The dashed lines are diffusion only cases under a no-flow condition.

Figure 10 shows the long-time dissolution kinetics presented by transient evolution of the particle size under (a) two sets of saturation conditions and various flow conditions, and (b) various particle inertias. As expected the saturation concentration $c_{0}$ defined at the interface of particle and solution medium is the primary factor that determines the dissolution rate. The dashed lines present the slowest dissolution kinetics under a no-flow condition. The oscillation frequency and displacement, and thus the bulk velocity, are likely to increase together during the orbital shaking process, which enhances the overall dissolution rate significantly from case (I) to (III). Curves that appear slightly wavy during the process are due to the oscillatory nature of mass transfer already observed previously 
in Fig. 9. The inertia or mass density effect on the dissolution kinetics of free particle suspended in oscillatory flow is shown in Fig. 10b, where saturation concentration is defined as $c_{0}=500 \mathrm{~kg} / \mathrm{m}^{3}$. Intuitively we know that higher inertia or higher $\lambda$ value gives higher relative velocity which would enhance the mass transfer and decrease the dissolution time. Figure 10b shows an opposite trend to this intuition because the characteristic dissolution time is essentially proportional to the mass density, given by Eq. (57), which dominates the evolution of the particle size during the dissolution process. The dashed line shown in Fig. $10 \mathrm{~b}$ is under a no flow condition. Thereby only a slightly increase of the dissolution rate or reduction of dissolution time due to the passive oscillatory motion for the given conditions. The result is consistent with the Sherwood number shown in Fig. 9b.

Summary Analytical and numerical studies are presented for the quantitative investigation of momentum, mass transfer, and dissolution kinetics of small colloidal particles in an oscillatory flow. The simplified model reveals basic characteristics of convective and diffusive transport involved in particle dissolution, and is very relevant to drug reconstitution driven by an orbital shaking process. Future investigations will extend the model to an three-dimensional free surface oscillatory flow and to a higher Reynolds number regime.

Acknowledgments We acknowledge partial financial support of this research from the National Science Foundation and Pfizer Inc.

[1] B. Abramzon and W.A. Sirignano, Droplet vaporization model for spray combustion calculations, Int. J. Heat Mass Transfer., 32(9), 1605-1618, 1989.

[2] K.C. Johnson, Dissolution and absorption modeling: model expansion to simulate the effects of precipitation, water absorption, longitudinally changing intestinal permeability, and controlled release on drug absorption, Drug Dev Ind Pharm., 29(8), 833-842, 2003.

[3] J. Landau, A. Dim, and R. Houlihan, A reciprocating-plate extraction column for hydrometallurgical applications, Metall. Trans., 4(12), 2827-2832, 1973. 
[4] E.S.R. Hassan, F. Mutelet, and J.Moïse, From the dissolution to the extraction of carbohydrates using ionic liquids, R. Soc. Chem. Adv., 3(43), 20219-20226, 2013.

[5] F.K. Crundwell, The dissolution and leaching of minerals: Mechanisms, myths and misunderstandings, Hydrometallurgy, 139(43), 132-148, 2013.

[6] B. Van Eerdenbrugh, L. Froyen, J. Van Humbeeck, J.A. Martens, P. Augustijns, and G.Van den Mooter, Drying of crystalline drug nanosuspensionsthe importance of surface hydrophobicity on dissolution behavior upon redispersion, Eur. J. Pharm. Sci., 35(1), 127-135, 2008.

[7] A.M. Al Taweel, M.I. Ismail, and M.Z. El-Abd, Effect of longitudinal vibrations on the masstransfer at vibrating electrodes, Chem. Ing. Tech., 46(20), 861, 1974.

[8] N.O. Lemcoff and G.J. Jameson, Hydrogenation of acetone in a vibrating slurry reactor, AIChE J., 21(4), 730-735, 1975.

[9] S.H. Zhang and E.J. Davis, Mass transfer from a single micro-droplet to a gas flowing at low Reynolds number, Chem. Eng. Comm., 50, 51-67, 1987.

[10] V. Pillay and R. Fassihi, Unconventional dissolution methodologie, J. Pharm. Sci., 88(9), 843-851, 1999.

[11] R.G. Rice and D.D. Do, Dissolution of a solid sphere in an unbounded, stagnant liquid, Chem. Eng. Sci., 61(2), 775-778, 2006.

[12] G. Bai, J.S. Bee, J.G. Biddlecombe, Q. Chen, W.T. Leach, Computational fluid dynamics (CFD) insights into agitation stress methods in biopharmaceutical development, Int. J. Pharm., 423, 264-280, 2012.

[13] Y. Wang, B. Abrahamsson, L. Lindfors, and J.G. Brasseur, Comparison and analysis of theoretical models for diffusion-controlled dissolution, Mol. Pharm., 9(5), 1052-1066, 2012.

[14] H. Thiermann, R. Schreiner, and P. Eyer, Dissolution kinetics of unstable drugs in twocompartment autoinjectors: Analysis of the individual shaking behaviour and influence of various shaking parameters on the dissolution rate of HI 6 in an automated system, Int. J. Pharm., 170(1), 23-32, 1998.

[15] A. Deicke and R. Süverkrüp, Dose uniformity and redispersibility of pharmaceutical suspensions I: quantification and mechanical modelling of human shaking behaviour, Eur. J. Pharm. Biopharm., 48(3), 225-232, 1999.

[16] J. Büchs, U. Maier, C. Milbradt, and B. Zoels, Power consumption in shaking flasks on rotary shaking machines: II. Nondimensional description of specific power consumption and flow 
regimes in unbaffled flasks at elevated liquid viscosity, Biotechnol. Bioeng., 68(6), 594-601, 2000 .

[17] W.A. Duetz and B. Witholt, Effectiveness of orbital shaking for the aeration of suspended bacterial cultures in square-deepwell microtiter plates, Biochem. Eng. J., 7(2), 113-115, 2001.

[18] M.J. Lighthill, Contributions to the theory of heat transfer through a laminar boundary layer, Proc. R. Soc. London, Ser. A, 202(1070), 359-377, 1950.

[19] R. Kronig and J. Bruijsten, On the theory of the heat and mass transfer from a sphere in a flowing medium at low values of Reynolds' number, Appl. Sci. Res., 2(1), 439-446, 1951.

[20] F.H. Garner and R.B. Keey, Mass-transfer from single solid spheres - I: Transfer at low Reynolds numbers, Chem. Eng. Sci., 9(2), 119-129, 1958.

[21] V.G. Levich, Physicochemical Hydrodynamics, Prentice-Hall Englewood Cliffs, NJ, 1962.

[22] A. Acrivos and T.D. Taylor, Heat and mass transfer from single spheres in Stokes flow, Phys. Fluids, 5(4), 387-394, 1962.

[23] S.K. Friedlander, A note on transport to spheres in Stokes flow, AIChE J., 7(2), 347-348, 1961.

[24] H. Brenner, Forced convection heat and mass transfer at small Péclet numbers from a particle of arbitrary shape, Chem. Eng. Sci., 18(2), 109-122, 1963.

[25] Y.P. Gupalo and Y.S. Ryazantsev, Mass and heat transfer from a sphere in a laminar flow, Chem. Eng. Sci., 27(1), 61-68, 1972.

[26] A.P. Burdukov and V.E. Nakoryakov, On mass transfer in an acoustic field, J. Appl. Mech. Tech. Phys., 6(2), 51-55, 1965.

[27] A.M. Al Taweel and J. Landau, Mass transfer between solid spheres and oscillating fluids - a critical review, Can. J. Chem. Eng., 54(5), 532-539, 1976.

[28] C.K. Drummond and F.A. Lyman, Mass transfer from a sphere in an oscillating flow with zero mean velocity, Comput. Mech., 6(4), 315-326, 1990.

[29] A. Saboni, S. Alexandrova, M. Karsheva, and C. Gourdon, Mass transfer from a contaminated fluid sphere, AIChE J., 57(7), 1684-1692, 2011.

[30] L.D. Landau and E.M. Lifshitz, Fluid Mechanics, Vol. 6. Course of Theoretical Physics, Pergamon Press, Oxford, 1987.

[31] R.B. Bird, W.E. Stewart, and E.N. Lightfoot, Transport Phenomena, John Wiley, New York, 1960. 
[32] J.Q. Feng, Diffusion-controlled quasi-stationary mass transfer for an isolated spherical particle in an unbounded medium, Chem. Eng. Comm., 200(1), 65-76, 2013.

[33] J.T. Carstensen and M. Dali, Determination of mass transfer dissolution rate constants from critical time of dissolution of a powder sample, Pharm. Dev. Technol., 4(1), 1-8, 1999.

[34] H. Tjong and H.-X. Zhou, Prediction of protein solubility from calculation of transfer free energy, Biophys. J., 95(6), 2601-2609, 2008.

[35] C. Pozrikidis, Introduction to Theoretical and Computational Fluid Dynamics, Oxford University Press, London, 1997. 